\title{
COMPOSTOS VOLÁTEIS E NOTA GLOBAL DOS ATRIBUTOS SENSORIAIS NA DISCRIMINAÇÃO DE VARIEDADES CLONAIS DE Coffea canephora COM GENÓTIPOS DE DIFERENTES CICLOS DE MATURAÇÃO
}

\author{
VOLATILE COMPOUNDS AND GLOBAL GRADE OF SENSORY \\ ATTRIBUTES IN THE DISCRIMINATION OF CLONAL VARIETIES OF Coffea \\ canephora WITH GENOTYPES FROM DIFFERENT MATURATION CYCLES
}

\author{
${ }^{1}$ Jéssica Louzada Machado \\ ${ }^{2}$ José Maria Rodrigues da Luz \\ ${ }^{1}$ Vanessa Moreira Osório \\ ${ }^{1}$ Adilson Vidal Costa \\ ${ }^{1}$ Tafarel Victor Colodetti \\ ${ }^{3}$ Lucas Louzada Pereira \\ ${ }^{2}$ Marliane de Cássia Soares da Silva \\ ${ }^{2}$ Tomás Gomes Reis Veloso \\ ${ }^{1 *}$ Marcelo Antonio Tomaz
}

\begin{abstract}
${ }^{1}$ Universidade Federal do Espírito Santo - Alegre ES - 29375-000. jessicalouzadamachado@yahoo.com.br; moreirava@yahoo.com.br; avcosta@hotmail.com; tafarelcolodetti@hotmail.com; ${ }^{2}$ Universidade Federal de Viçosa, Departamento de Microbiologia, Avenida PH Rolfs S/N Viçosa, Minas Gerais-Mg 36570-000, Brasil, mcassiabio@gmail.com; josemarodrigues@yahoo.com.br; tomasgomesrv@gmail.com ${ }^{3}$ Instituto Federa do Espírito Santo- Campus Venda Nova do Imigrante - Coffee Design lucas.pereira@ifes.edu.br *Autor de correspondência
\end{abstract}

Artigo submetido em 14/112021, aceito em 30/11/2021 e publicado em 30/12/2021.

Resumo: A obtenção de genótipos de Coffea canephora pelo melhoramento genético tem contribuído para aumento da produtividade e qualidade sensorial da bebida. A uniformidade do ciclo de maturação é um importante fator considerado no desenvolvimento de variedade clonais. Assim, o objetivo desse estudo foi avaliar a discriminação química e sensorial de três variedades clonais de $C$. canephora com genótipos de diferentes ciclos de maturação dos frutos baseada na composição de compostos voláteis e na nota global dos atributos sensoriais. As amostras foram constituídas por 54 frutos cerejas, sendo 18 frutos de cada variedade clonal (9 genótipos por ciclo de maturação e 2 repetições). Os frutos foram secados, torrados e moídos para realização das análises químicas e sensoriais. A extração, a caracterização e a identificação dos compostos voláteis foram realizadas por micro extração em fase sólida combinada à cromatografia gasosa e a espectrometria de massas. Para análise sensorial foi utilizado o protocolo da Associação Americana de Cafés Especiais. Nos frutos dos diferentes genótipos foram identificados 4 classes e 12 diferentes compostos voláteis relacionados aos atributes sensoriais. 
A análise multivariada de coordenadas principais mostrou que os clones de maturação precoce estão mais afastados dos genótipos de maturação intermediaria que daqueles de maturação tardia em relação a composição de compostos voláteis. Entretanto, as variedades clonais tiveram notas globais dos atributos sensoriais da bebida similares. Portanto, há uma variabilidade genética entre as variedades clonais, entretanto a discriminação desses genótipos baseada nas notas globais deve ser investigada considerando a contribuição individual dos atributos sensoriais.

Palavras-chave: Cultivar; melhoramento genético; qualidade sensorial; Conilon; Kovats.

Abstract: Obtaining Coffea canephora genotypes through genetic improvement has contributed to increased productivity and sensory quality of the beverage. The uniformity of the maturation cycle is an important factor considered in the development of clonal varieties. Thus, the objective of this study was to evaluate the chemical and sensory discrimination of three clonal varieties of $C$. canephora with genotypes of different fruit maturation cycles based on the composition of volatile compounds and in the global score of the sensory attributes. The samples were 54 cherry fruits with 18 fruits of each clonal variety ( 9 genotypes per maturation cycle and 2 replications). The fruits were dried, roasted and ground to carry out chemical and sensory analyses. The extraction, characterization and identification of volatile compounds were performed by solid phase microextraction combined with gas chromatography and mass spectrometry. For sensory analysis, the protocol of the American Specialty Coffee Association was used. In the fruits of different genotypes, 4 classes and 12 different volatile compounds related to sensory attributes were identified. The multivariate analysis of principal coordinates showed that early maturing clones are farther away from intermediate maturing genotypes than from late maturing ones in relation to volatile compound composition. However, the clonal varieties had similar overall ratings of the beverage's sensory attributes. Therefore, there is a genetic variability among clonal varieties, however the discrimination of these genotypes based on global scores must be investigated considering the individual contribution of sensory attributes.

Keywords: Grow crops; genetical enhancement; sensory quality; Conilon; Kovats. 


\section{INTRODUÇÃO}

A cafeicultura é uma atividade agrícola mundial que contribui substancialmente no crescimento sócio, econômico e cultural de mais de 70 países, sendo os países subdesenvolvidos ou em desenvolvimento os principais produtores e exportadores para os países desenvolvidos, que são os maiores consumidores da bebida (FRANCK et al., 2016; SILVA, 2017).

O comércio marítimo realizado durante as Grandes Navegações dos países Europeus no século XV e XVI contribuiu para a difusão da atividade cafeeira no continente americano (TAUNAY, 1945). A origem geográfica da biodiversidade da planta do café está localizada no continente Africano de onde foi obtido as sementes para reprodução em outras regiões do mundo que contribuiu para o aparecimento de novas variedades genéticas (BEENHOUWER et al., 2015; FERRÃO et al., 2017a; SOUZA et al., 2015). Além disso, o consumo da bebida do café teve início no século IX na África, se popularizou no século XVI na Ásia e nos séculos posteriores tornou-se umas das bebidas mais consumidas no mundo (MARTINS, 2012).

A produção de grãos de café advém especificamente do gênero Coffea que possui cerca de 124 espécies descritas com destaque para Coffea arabica e Coffea canephora que tem uma grande importância comercial (DAVIS et al., 2011). Essa diversidade de espécies está dividida por meio das diferentes regiões de cultivos, fatores edafoclimáticos e genótipos e fenótipos distintos de variedades botânicas (FERRÃO et al., 2017b).

No Brasil, o cultivo de C. arabica concentra cerca de $85 \%$ da produção nacional nos estados de Minas Gerais, São Paulo, Espírito Santo e Bahia, enquanto, o C. canephora, $95 \%$ da produção nacional advém do Espírito Santo, Bahia e Rondônia (BRASIL, 2018). O país é o maior produtor e exportador mundial de café, e segundo maior consumidor do produto. O parque cafeeiro nacional é estimado em 1,74 milhões de hectares $(80,7 \%)$ com o cultivo de café arábica e 415,9 mil (19,03\%) hectares com plantio de café Conilon (CONAB, 2019). Além disso, a cafeicultura é a principal atividade agrícola do Espírito Santo e está presente em quase todos os municípios desse estado (INCAPER, 2012).

A demanda por cafés de qualidade estimula estudos e avaliações que buscam expressar os atributos intrínsecos sobre a qualidade do café, provenientes de interações entre o ambiente de produção e as características genéticas da espécie e variedades clonais (ANDROCIOLI et al., 2003; LEITE, 1991).

As cultivares de C. canephora compõem-se de variedades clonais e variedades sintéticas (FERRÃO et al., 2017a). Em 2019, haviam dezesseis cultivares clonais registradas no Ministério da Agricultura e Abastecimento (FERRÃO et al., 2019). Dez desses cultivares, a exemplo de Diamante ES8112, Jequitibá ES8122 e Centenária ES8132 foram desenvolvidas pelo Instituto Capixaba de Pesquisa, Assistência Técnica e Extensão Rural (INCAPER) com o objetivo de aumentar a produtividade dos frutos e a qualidade sensorial da bebida do café regulando geneticamente $\mathrm{o}$ ciclo de maturação dos frutos.

A uniformidade de maturação dos frutos é uma frequente preocupação da cafeicultura e um dos fatores principais considerados no melhoramento de variedades de $C$. canephora (CORRÊA et al., 2015; ROCHA et al, 2015; MARCOLAN \& ESPÍNDULA, 2015). A ocorrência dessa desuniformidade compromete a colheita e tem reflexões sobre a qualidade final do produto (ROCHA et al., 2015).

O ciclo de maturação representa o intervalo entre a floração e a colheita dos frutos (CORREA et al., 2015; ROCHA et al., 2015). De acordo com Rocha et al. (2015), a florada é observada entre 30 e 120 dias após a colheita e as maturações precoce e intermediária ocorre, respectivamente 
após 90 e 270 dias da florada principal. Assim, a maturação tardia tem um período ocorrência superior a esses intervalos descritos. Além disso, aumento na atividade respiratória, síntese de etileno, metabolismo de açúcares e ácidos, degradação da clorofila e a síntese de pigmentos são características bioquímicas e fisiológicas observadas durante maturação dos frutos do cafeeiro (CORREA et al., 2015).

A qualidade do café está relacionada ao um conjunto de atributos físicos, químicos e sensoriais que são influenciados por múltiplos fatores genéticos, culturais, ambientais, colheita, ciclo ou grau de maturação, tipo de processamento, processos de secagem, armazenamento; torração e preparo da bebida (ALVARENGA, 2017; MONDELLO, 2005; PEREIRA, 1997; PEREIRA et al., 2020).

Na composição química dos grãos crus de café, os compostos nitrogenados, os açúcares e os ácidos clorogênicos são precursores de compostos responsáveis pelo aroma e sabor da bebida (FLAMENT, 2002). Essa transformação química ocorre durante a torração, dos grãos (RIBEIRO et al., 2009). Em adição, na formação do sabor cerca de trezentos compostos químicos dos grãos crus originam quase mil constituintes voláteis que incluem as classes de furanos, pirazinas, cetonas, fenóis, ácidos, aldeídos, compostos sulfurados e piridinas (TOCI et al., 2014). Além disso, fatores genéticos da planta podem influenciar e contribuir na composição química dos precursores do sabor no café. As variedades genéticas de $C$. canephora apresentam teores distintos de cafeína, trigonelina, ácidos clorogênicos e sacarose (AGUIAR et al., 2005; PEREIRA et al., 2021).

A determinação da qualidade da bebida de café é realizada, mundialmente, pela análise sensorial (PEREIRA et al., 2018). Criada na segunda metade do Século $\mathrm{XX}$, essa a análise é uma ciência relativamente nova, na qual busca padronizar a qualidade sensorial dos produtos (LAWLESS \& HEYMANN,
2010). As metodologias de análise sensorial são realizadas por degustadores treinados, no qual permite por meio das funções de sentido do gosto, olfato e tato a sensibilidade, para perceber a diferença sensoriais entre as amostras (MARQUES, 2017). Essa degustação realizada por provadores profissionais é denominada de "prova da xícara", por meio de Q-Graders (PEREIRA et al., 2018).

Para a análise sensorial de cafés conilon é utilizado o Protocolo de Degustação de Robustas Finos, no qual as amostras de cafés crus são preparadas e torradas com 24 horas de antecedência, permanecendo em descanso de 8 horas após a torra para a moagem (PEREIRA et al., 2018; Machado, 2019). O objetivo do teste de degustação é avaliar a qualidade dos 10 atributos sensoriais: Fragrância/Aroma, Uniformidade, Ausência de Defeitos (Xícara Limpa), Doçura, Sabor, Acidez, Corpo, Finalização, Equilíbrio e Defeitos que são classificados por uma escala numérica centesimal (PEREIRA et al.; 2016; UCDA, 2012). Além disso, segundo Pereira et al. (2018), os princípios éticos e normativos, a exemplo da imparcialidade do pesquisador, a amostragem aleatória e a casualização dos tratamentos e das repetições dos experimentos científicos compõem as análises sensoriais.

Nesse contexto, devido as complexidades entres os fatores ambientais, genéticos, agronômicos, processamento pós-colheita, armazenamento e torrefação que determinam a qualidade do café são necessários mais estudos para avaliar as correlações de cultivares com a qualidade da bebida de café (BRIOSCHI JUNIOR et al., 2020; DE BRUYN et. al., 2017; PEREIRA et al., 2021). Além disso, há poucos estudos que avaliam os constituintes químicos e a correlação desses compostos com as características sensoriais da bebida de café Conilon.

Assim, o objetivo desse estudo foi avaliar a discriminação química e sensorial de três variedades clonais de Coffea canephora com genótipos de diferentes 
ciclos de maturação dos frutos baseada na composição de compostos voláteis e nos atributos sensoriais.

\section{MATERIAL E MÉTODOS}

Esse estudo foi realizado no Centro de Ciências Exatas, Naturais e da Saúde da Universidade Federal do Espírito (campus Alegre) em parceira com Instituto Capixaba de Pesquisa, Assistência Técnica e Extensão Rural (Incaper) e com Laboratório de Análise e Pesquisa em Café (LAPC) do Instituto Federal do Espírito Santo, campus Venda Nova do Imigrante.

\subsection{LOCAL DO EXPERIMENTO E OBTENÇÃO DAS AMOSTRAS}

A coleta das amostras constituídas de grãos de café foi realizada no campo experimental do Incaper localizado numa fazenda no município de Alegre, ES conforme as coordenadas geográficas $20^{\circ}$ 52' 07'S, 41 ${ }^{\circ} 28$ ' 43”'W e $647 \mathrm{~m}$ de altitude. Esse campo foi implantado em 2015 com o plantio de 27 genótipos de C. canephora sendo nove mudas de cada um dos cultivares clonais que apresentam diferença em relação ao ciclo de maturação (Quadro $1)$.

As plantas foram cultivadas no espaçamento de 3,0 x 1,0 m, com três ramos ortotrópicos e poda programada para o ciclo reprodutivo do café Conilon.

Quadro 1: Cultivares clonais de 27 genótipos de Coffea canephora com diferentes ciclos de maturação dos frutos

\begin{tabular}{|c|c|c|}
\hline $\begin{array}{c}\text { Cultivar } \\
\text { clonal }\end{array}$ & $\begin{array}{c}\text { Quantidade } \\
\text { de } \\
\text { genótipos }\end{array}$ & $\begin{array}{c}\text { Ciclos de } \\
\text { maturação }\end{array}$ \\
\hline $\begin{array}{c}\text { Diamante } \\
\text { Incaper 8112 }\end{array}$ & 9 & Precoce \\
\hline $\begin{array}{c}\text { Jequitibá } \\
\text { Incaper 8122 }\end{array}$ & 9 & Intermediária \\
\hline $\begin{array}{c}\text { Centenária } \\
\text { Incaper 8132 }\end{array}$ & 9 & Tardia \\
\hline
\end{tabular}

Fonte: INCAPER (2012), Machado (2019)

O campo experimental segue delineamento de blocos casualizados com seis plantas por genótipos (Quadro 1) e as parcelas experimentais foram compostas por amostras (6 litros) de grãos obtidas em duas plantas de cada genótipo. Essa amostragem foi realizada na colheita entre julho a setembro de 2018.

A retirada dos grãos foi iniciada quando $85 \%$ dos frutos atingiam o estágio de Cereja (maduro). Os seis litros de amostras foram submetidos ao processamento por via seca. Os frutos foram secados em terreiro com exposição solar até cerca de 35\% (b.u.) de umidade e em uma estufa de circulação forçada de ar até atingir $12 \%$ b.u. Em seguida, os grãos foram torrados e moídos para realização das análises químicas e sensoriais.

2.2. EXTRAÇÃO, DETERMINAÇÃO E IDENTIFICAÇÃO DE COMPOSTOS VOLÁTEIS DOS GRÃOS DE CAFÉ

A micro-extração em fase sólida no modo headspace combinado com cromatografia gasosa acoplada à espectrometria de massas foi utilizada para obtenção do perfil de compostos voláteis das amostras de café Conilon torradas e moídas. Os procedimentos de extração, determinação e identificação dos compostos voláteis utilizando $1 \mathrm{~g}$ das amostras foi realizada conforme descrito por Machado (2019). Para identificação dos compostos voláteis uma mistura de hidrocarbonetos com cadeia de 8 (C8) a 23(C23) átomos de carbono injetados nas mesmas condições cromatográficas das amostras foi utilizada. A identificação desses compostos foi realizada pela comparação entre os espectros das amostras, dos hidrocarbonetos de referências (C8 a C23) e dos compostos da biblioteca (WILEY7V100) do espectrofotômetro de massa. A quantificação dos compostos voláteis foi realizada utilizando o índice de Kovats (KOVÀTS, 1958; QU et al., 2021). 2.3. ANÁLISES SENSORIAIS DA BEBIDA

As análises sensoriais foram realizadas utilizando o protocolo da Associação Americana de café especiais com seis julgadores, Q-Grader, credenciados (Pereira et al., 2018; SCAA, 2013). 
Cinco xícaras de cada amostra dos 27 genótipos foram preparadas para degustação dos 6 Q-grader. No preparo da bebida foram utilizados $8,25 \mathrm{~g}$ de café torrado e moído em $150 \mathrm{~mL}$ de água com ponto de infusão entre $92-95^{\circ} \mathrm{C}$ (Machado, 2019).

Os Q-Grader utilizaram uma sequência para a degustação das amostras e individualmente registraram as notas e as observações. A avaliação da bebida iniciouse pela fragrância exalada pelo pó ainda seco. Após a adição da água quente, as amostras foram avaliadas quanto ao aroma e, após a redução da temperatura para aproximadamente $55-60^{\circ} \mathrm{C}$, os outros atributos sensoriais (sabor, acidez, amargor/doçura, equilíbrio, sensação na boca, retrogosto, uniformidade, limpeza, conjunto e nota global) foram avaliadas. A bebida foi analisada por sucção, cobrindose a maior área da cavidade bucal e a nota global foi calculada pela soma das notais individuais de cada atributo sensorial investigado.

\subsection{ANÁLISES ESTATÍSTICAS}

Para avaliar as diferenças no perfil de compostos voláteis nos três grupos de genótipos com diferentes ciclos de maturação (precoce, intermediário e tardio), uma análise de coordenadas principais (PCoA) a distância de Bray-Curtis foi realizada. O pacote vegan v. 3.3 .5 (OKSANEN et al., 2020) foi utilizado para calcular a matriz de distâncias e a função cmdscale foi utilizada para realizar a redução de dimensionalidade. A análise de permutação de variância (PERMANOVA) foi realizada com a função adonis do pacote Vegan. Todas análises foram realizadas no programa R version 4.1 .2 (2021-11-01) usando as bibliotecas base (R CORE TEAM, 2021).

As correlações de Spearman foram calculadas usando o pacote Hmisc v. 4.5.0. A confecção dos gráficos foi realizada utilizando a biblioteca ggplot2 v. 3.3 .5 (WICKHAM, 2009).

\section{RESULTADOS E DISCUSSÃO}

Nos frutos dos diferentes genótipos foram identificados 4 classes e 12 diferentes compostos voláteis relacionados aos atributos sensoriais do café (Tabela 1). A intensidade desses compostos variou em função do ciclo de maturação. Uma predição utilizando cromatografia gasosa acoplada a espectrofotometria de massa (GS-MS) mostrou a presença de 29 compostos voláteis, a exemplo de piridina, 2-metiltetrahidrofuran-3-ona, 2,5-dimetil3(2H)furano, 5-metilfurfural, 2,5-dimetil-3etilpirazina, 2-acetil-3-metilpirazina, 5hidroximetilfuraldeido e 4-etenil-2metoxifenol em 40 amostras de frutos de café arábica com $85 \%$ de grau de maturação (AGNOLETTI et al. (2022). A espécie de café e a sensibilidade das técnicas analíticas utilizado por Agnoletti et al. (2022) e neste estudo explicam na diferença na quantidade de compostos voláteis observadas. No entanto, o perfil químico dos frutos verde de C. canephora determinado de GS-MS mostrou a identificação de 28 compostos voláteis (TANG et al., 2021). Em frutos cereja de café Conilon de diferentes altitudes de plantio submetidos ao método de processamento seco e torrefação foram observados 36 compostos voláteis (PEREIRA et al., 2021). Assim, a composição de compostos voláteis de frutos de café depende de vários fatores, a exemplo de variedades vegetais, regiões de cultivo, altitudes, grau de maturação e processamento pós-colheita.

Os genótipos de maturação precoce tiveram maior proporção relativa de metilpirazina, enquanto os furanos e fenóis estão, respectivamente, com maiores proporções nos genótipos de maturação intermediária e tardia (Tabela 1). Todavia, a diferença na composição de compostos voláteis em função do ciclo de maturação tem sido poucos discutidos em estudos científicos que utilizam amostras de café verde ou com grau de maturação de 85 a 90 $\%$ nos experimentos (AGNOLETTI et al., 2022; DEBONA et al., 2021; EVANGELISTA et al., 2015; MALTA et al., 2020). 
Tabela 1: Composição de compostos voláteis identificados em três variedades clonais de Coffea canephora com genótipos diferentes ciclos de maturação dos frutos e características sensoriais dos doze compostos químicos desses genótipos

\begin{tabular}{|c|c|c|c|c|c|}
\hline \multirow[t]{3}{*}{ Classe } & \multirow[t]{3}{*}{ Composto } & \multirow[t]{3}{*}{ Características sensoriais* } & \multirow{2}{*}{\multicolumn{3}{|c|}{$\begin{array}{c}\text { Presença e intensidade relativa dos } \\
\text { compostos voláteis** }\end{array}$}} \\
\hline & & & & & \\
\hline & & & Precoce & Intermediária & Tardia \\
\hline \multirow{3}{*}{ Furano } & Furfural & Amêndoas/ caramelo & ++ & +++ & + \\
\hline & 2-Furil-metanol & Doce/ caramelo/nozes & ++ & +++ & + \\
\hline & 5-Metilfurfural & Doce/caramelo/ amêndoa/ Frutado & ++ & +++ & + \\
\hline \multirow{6}{*}{ Pirazina } & Metilpirazina & Nozes/ chocolate/ torrado & +++ & ++ & + \\
\hline & 2-Etilpirazina & Manteiga/torrado & ++ & ++ & + \\
\hline & 2.5-Dimetilpirazina & Nozes/gramado/milho/torrado & ++ & ++ & + \\
\hline & 2.6-Dimetilpirazina & Nozes/verde/ torrado & ++ & ++ & + \\
\hline & 2.3-Dimetillpirazina & Nozes/verde/ torrado & ++ & + & ++ \\
\hline & 2-Acetil-3metilpirazina & Batata doce/ nozes & + & + & ++ \\
\hline \multirow{2}{*}{ Fenol } & 2-Metoxifenol (guaiacol) & Especiarias/fenólico & + & ++ & +++ \\
\hline & 4-Etenil-2-metoxifenol & Especiarias/fenólico & + & ++ & +++ \\
\hline Piridina & Piridina & $\begin{array}{c}\text { Adstringente/ amargo/ } \\
\text { queimado/torrado }\end{array}$ & + & + & + \\
\hline
\end{tabular}

Fonte: Autores (2021). *Agnoletti et al. (2022). **Os sinais de soma e quantidade deles representam, respectivamente, a presença e quantidade dos compostos voláteis nas amostras. As cores destacam os compostos com maior intensidade em cada período de maturação. 
Em estudo sobre a discriminação genética de genótipos utilizando o perfil dos compostos voláteis e o painel dos atributos sensoriais foi observado uma diferença na concentração relativa dos furanos entre as variedades clonais Diamante, Jequitibá e Centenária (MACHADO, 2019). O autor concluiu que a formação desses compostos varia com grau de maturação dos frutos. A presença de furanos indica ganhos significativos para a qualidade da bebida do café com atribuição de características sensoriais (Tabela 1) de sabor doce e semelhante ao sabor de frutas e castanhas (nozes) ou caramelo (FLAMENT, 2002). Além disso, os genótipos com menor duração do ciclo de maturação dos frutos apresentam maior velocidade de acúmulo de massa de matéria seca e nutrientes (MACOLAN et al., 2015) que pode explicar a diferença na composição de compostos voláteis nos genótipos de maturação precoce em relação aos genótipos de outros tipos de maturação.

A composição de frutose nos frutos de café também tem influência sobre o teor de furanos devido esse monossacarídeo apresentar na conformação cíclico um anel furanosídio. Os furanos são formados pela degradação de glicídios, no entanto, há evidências que alguns furanos podem ser formados pela degradação de terpenos e polissacarídeos (FLAMENT, 2002). Bressanello et al. (2017) descrevem a formação de furanos a partir da degradação térmica dos açúcares, mas a presença de outros grupos funcionais tende a aumentar a complexidade sensorial desses compostos. Segundo Correa et al. (2015), semelhante aos outros componentes químicos, o teor de açúcar nos grãos de café pode diferir por causa das variações de cultivares e estádios de maturação, bem como as condições climáticas e as características da região produtora. Além disso, os furanos são precursores de compostos que produzem a percepção odorífica de caramelo
(FLAMENTE, 2002) e se tratando de compostos furânicos, o furfural, 5metilfurfural e furfuril acetato são considerados marcantes para o aroma do café (AKIYAMA et al., 2005). Entretanto, a concentração desses compostos em café torrado pode definir a contribuição positiva e negativa para as características sensoriais da bebida (MOREIRA et al., 1999).

As pirazinas apresentam aroma doce que é uma característica importante para discriminação sensorial da bebida do café (MACHADO et al., in press). Cerca de 100 compostos da classe das pirazinas estão presentes no café torrado, mas apenas algumas delas, a exemplo de 2-etilpirazina, 2-etil-3,5-dimetilpirazina, 2,5dimetilpirazina, 2,6-dimetilpirazina, 2metilpirazina e 2,3-dimetilpirazina são consideradas marcantes para o aroma da bebida de café (AKIYAMA et al., 2005, SANZ et al., 2002). Essa classe de compostos voláteis teve maiores teores nas variedades de maturação precoce e intermediária que na tardia mostrando ser importante na discriminação química de genótipos de café, principalmente aqueles genótipos de maturação precoce devido aos teores de metilpirazina (Tabela 1).

Os fenóis foram importantes na discriminação química dos genótipos de maturação tardia em relação aos outros genótipos de maturação precoce e intermediária (Tabela 1). O tipo e a concentração de compostos fenólicos em café torrados dependem da variedade, estádios de maturação, processamento póscolheita e condições de armazenamento e torrefação (PEREIRA et al., 2021; TANG et al., 2021, WANG et al., 2021,). Os fenóis voláteis do café apresentam características sensoriais bem variadas (AGNOLETTI et al., 2022; MACHADO, 2019; PEREIRA et al., 2018). O 2-metoxifenol (guaiacol) e o 4etenil-2-metoxifenol apresentam independente da concentração um perfil sensorial importante para o café torrado 
(MACHADO, 2019; MOREIRA et al., 1999). Em adição, o 4-etenil-2-metoxifenol foi o composto volátil mais importante na discriminação química e sensorial de 27 genótipos de $C$. canephora (MACHADO et al., in press). Para Machado (2019), a concentração de fenol é diretamente proporcional a qualidade da bebida do café. Esses resultados mostram a importância dos compostos fenólicos para a qualidade sensorial da bebida do café.

A piridina teve baixa contribuição na diferenciação química dos genótipos de diferentes ciclos de maturação (Tabela 1). Semelhantes aos compostos das classes das pirazinas e dos furanos, a piridina também é produto da degradação térmica de trigonelina, aminoácidos e os açucares (TANG et al., 2021). Nesse caso, as condições térmicas da torrefação dos grãos podem também ser importante na composição de compostos voláteis na bebida do café. Além disso, a piridina tem sabor picante e contribui para um odor amoniacal desagradável no café torrado (MACHADO, 2019). Esse autor acrescenta que o desenvolvimento de estudos sobre aspectos sensoriais e contribuições das piridinas para a qualidade sensorial dos grãos de café torrados pode explicar a baixa variação nos teores de desses compostos entre nas variedades clonais de $C$. canephora.

Os genótipos de maturação precoce tiveram composições químicas distintas dos genótipos de maturação intermediária e tardia (Figura 1).

Figura 1: Análise de coordenadas principais (PCoA) e PERMANOVA da composição de compostos voláteis de genótipos de Coffea canephora com diferentes períodos de maturação dos frutos. Pontos (azul, verde e vermelho) representam os noves genótipos individuais. Os triângulos e as elipses (com as mesmas cores dos pontos, mas com tonalidade clara) indicam, respectivamente a posição do centróide (mediana) e o intervalo de confiança de 95 \% para cada variedade clonal (Quadro 1).
Os valores nos eixos da abscissa e coordenada representam o percentual de explicação de cada eixo.

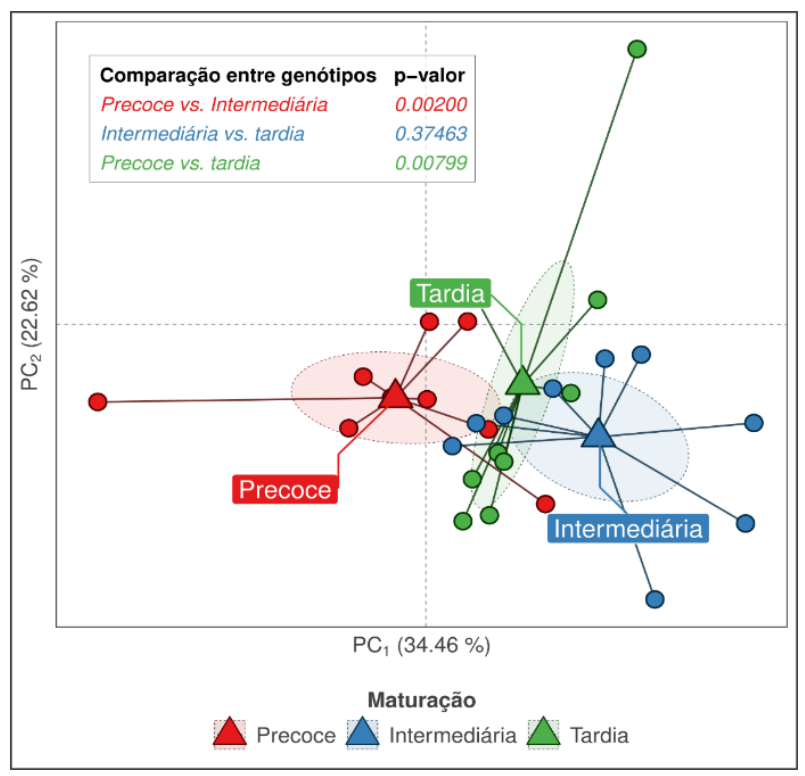

Fonte: Autores (2021)

No perfil da figura 1 pode ser observado ainda que os clones de maturação precoce estão mais afastados dos genótipos de maturação intermediaria $(\mathrm{p}=0.002)$ que daqueles de maturação tardia $(\mathrm{p}=0.00799)$. Por outro lado, os genótipos de maturação intermediária e tardia não apresentam diferenças significativa entre si em relação a composição dos compostos voláteis, uma vez que o $p$-valor $(0,37463)$ foi maior que 0,05. Assim, o ciclo de maturação promove alterações na composição química dos frutos do café que pode ter reflexos nos teores de compostos voláteis dos grãos (Figura 1, tabela 1) e nos atributos sensoriais da bebida (Tabela 2) como observado nos resultados desse estudo utilizando variedades clonais de diferentes ciclos de maturação dos frutos de $C$. canephora. 
Tabela 2: Nota Global dos atributos sensoriais da bebida de café dos genótipos de Coffea canephora com diferentes períodos de maturação.

\begin{tabular}{lccc}
\hline Maturação & Nota-Global & $\begin{array}{l}\text { Descrição de } \\
\text { qualidade }\end{array}$ & Classificação* $^{*}$ \\
\hline Precoce & $77,86 \pm 0,83$ & Muito bom & Prêmio \\
Intermediária & $78,09 \pm 1,25$ & Muito bom & Prêmio \\
Tardia & $78,80 \pm 0,87$ & Muito bom & Prêmio \\
\hline
\end{tabular}

Fonte: Autores (2021). UCDA (2012)

Figura 2: Correlação entre a nota final da bebida de café de três variedades clonais de Coffea canephora com genótipos de diferentes períodos de maturação e os compostos voláteis desses genótipos. $\mathrm{r}=$ correlação de Pearson. $\mathrm{p}=\mathrm{p}$-valor. Asterisco = modelo matemático significativo com um nível de significância de 0.05 .

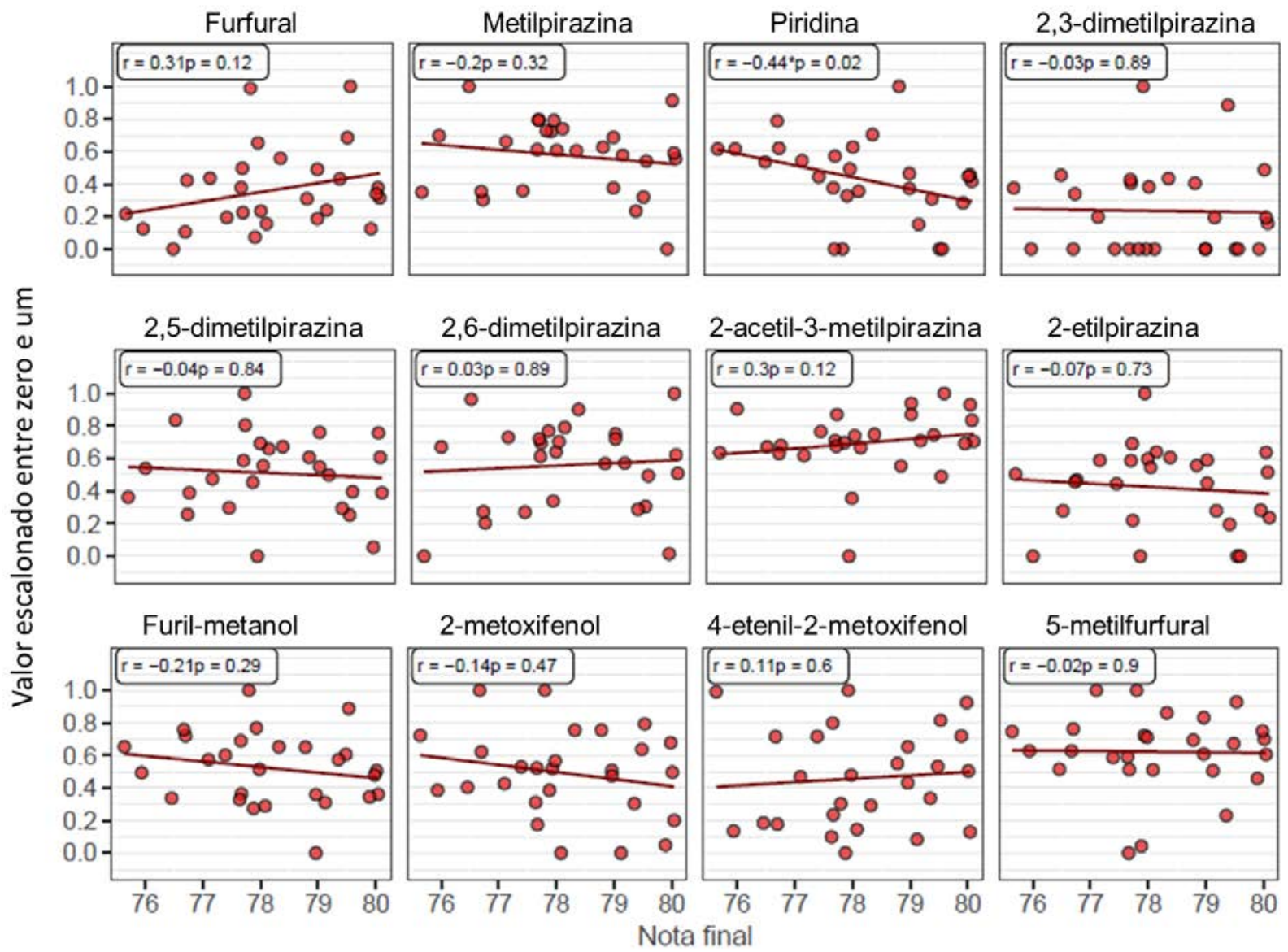

Fonte: Autores (2021) 
Em adição, Bragança et al. (2001) reforçam que cada estágio de desenvolvimento do fruto de café possui funções fisiológicas e metabólicas próprias que são essenciais para qualidade final dos grãos de café.

$$
\text { Apesar da composição de }
$$
compostos voláteis da variedade clonais de maturação precoce tem sido diferente das outras variedades (Figura 1), elas tiveram notas globais dos atributos sensoriais da bebida similares com média superior a 77 pontos (Tabela 2).

A qualidade sensorial da bebida do café depende do equilíbrio da composição química dos grãos torrados com contribuições sensoriais de diferentes compostos (PEREIRA et al., 2020, DEBONA et al., MALTA et al., 2020). Segundo Machado (2019), um único composto não pode ser considerado um marcador de qualidade para a bebida de café e todos os atributos sensoriais da bebida de genótipos de $C$. canephora, com destaque para retrogosto e sabor apresentam correlação positiva com a nota global. Assim, as variedades clonais com genótipos de diferentes maturações podem apresentar distintos percepção de aromas, sabor e palatabilidade na bebida do café (Tabela 1).

Com exceção da piridina que teve uma correlação negativa significativa com a nota geral dos atributos sensoriais, nenhuma outra correlação significativa foi observada entre os compostos voláteis dos genótipos e as notas gerais (Figura 2). Essa falta dessa correlação pode ser devido a análise estatística ter sido feita com a soma dos atributos sensoriais (nota global), uma vez que os compostos voláteis do café apresentam características sensoriais distintas (Tabela 1).

A nota global não foi utilizada no estudo da divergência genética entre os genótipos de Conilon devido ser oriunda do somatório dos atributos sensoriais e acarretaria a maior parte da contribuição relativa para si (MACHADO, 2019, MACHADO et al., in press). Assim, Machado (2019) mostrou que o sabor, a sensação na boca, o amargor/doçura, o retrogosto e a fragrância/aroma foram os atributos sensoriais com maior contribuição para a nota global da bebida de café. A análise individual dos atributos sensoriais fragrância/aroma, acidez, corpo e balanço apresentam diferentes coeficientes de correlação com a composição de compostos voláteis do café arábica (AGNOLETTI et al., 2022). Esses autores mostraram ainda que 15 compostos voláteis são responsáveis pela predição das notais sensoriais e refletem diretamente na qualidade da bebida. Em adição, as bebidas preparadas com os cafés dos genótipos analisados tiveram notas gerais (Tabela 2) para descrição de qualidade como muito bom e classificação como um tipo de café prêmio segundo os critérios da SCAA (2013).

\section{CONCLUSÕES}

O ciclo de maturação tem influência sobre a composição de compostos voláteis do café conilon. Os genótipos de maturação precoce apresentam composição de compostos voláteis distintas dos outros genótipos de ciclos de maturação intermediária e tardia. Portanto, há uma variabilidade genética entre as variedades de C. canephora com genótipos de diferentes ciclos de maturação para o perfil de compostos voláteis, entretanto a discriminação desses genótipos baseada nas notas globais dos atributes sensoriais deve ser investigada considerando a contribuição individual de cada um dos atributos sensoriais.

\section{AGRADECIMENTOS}

Agradecimentos a FAPES e CAPES,
CNPQ.

\section{REFERÊNCIAS}

AGNOLETTI, Bárbara Zani et al.

Multivariate calibration applied to study of volatile predictors of arabica coffee

quality. Food Chemistry, v. 367, p.

130679, 2022.

https://doi.org/10.1016/j.foodchem.2021.1 30679 
AGUIAR, A. T. E. Descritores para caracterização de cultivares e linhagens de café tipo arábica. 2001. 98p. Dissertação (Mestrado em Melhoramento Genético Vegetal) - Universidade Estadual de Campinas, Campinas, 2001.

AKIYAMA, Masayuki et al. Characterization of flavor compounds released during grinding of roasted robusta coffee beans.

Food Science and Technology Research, Switzerland, v. 11, n. 3, p. 298-307, 2005

ALVARENGA, S. T. Caracterização química e sensorial de cafés especiais do sul de

BEENHOUWER, Matthias De et al. Changing soil characteristics alter the arbuscular mycorrhizal fungi communities of Arabica coffee (Coffea arabica) in Ethiopia across a management intensity gradient. Soil Biology and Biochemistry, 91:133-139, 2015. https://doi.org/10.1016/j.soilbio.2015.08.0 37.

BRAGANÇA, S. M., et al. Variedades clonais de café Conilon para o Estado do Espírito Santo. Pesquisa Agropecuária Brasileira, v. 36, n. 5, p. 765-770, 2001.

BRASIL. Política Agrícola. Café no Brasil. Ministério da Agricultura, Pecuária e Abastecimento. Disponível em: $<$ http://www.agricultura.gov.br/assuntos/p oliticaagricola/cafe/cafeiculturabrasileira>. Acessado em: 08 de novembro de 2021.

BRESSANELLO, Davide et al. Coffee aroma: Chemometric comparison of the chemical information provided by three different samplings combined with GC-MS to describe the sensory properties in cup.

Food Chemistry, v.214, p. 218-226, 2017. BRIOSCHI JUNIOR Derio et al. ().

Microbial fermentation affects sensorial, chemical, and microbial profile of coffee under carbonic maceration. Food Chemistry, v. 342, p. 128296, 2020https://doi.org/10.1016/j.foodchem.20 20.128296

CONAB - Companhia Nacional de Abastecimento. Levantamento de safras, Janeiro. 2019. Disponível em: <https://www.conab.gov.br/infoagro/safras $>$.
Minas Gerais. 2017. 120p. Tese

(Doutorado em Ciência dos Alimentos) Programa de Pós Graduação em Ciência dos Alimentos, Universidade Federal de Lavras, Lavras, 2017.

ANDROCIOLI, Armando et al.

Caracterização da qualidade de bebida dos cafés produzidos em diversas regiões do Paraná. In: SIMPÓSIO DA PESQUISA DE CAFÉS DO BRASIL, 3., 2003, Porto Seguro. Anais.. Brasília, DF: Embrapa Café, p. 256-257, 2003.

CORRÊA, Paulo César et al. Propriedades físicas e químicas interferentes na póscolheita do café. In: MARCOLAN, A. L.; ESPINDULA, M. C. Café na Amazônia. 1. ed. Brasília, DF:EMBRAPA Rondônia, 2015.

DAVIS, Aaron et al. Growing coffee: Psilanthus (Rubiaceae) subsumed on the basis of molecular and morphological data; implications for the size, morphology, distribution and evolutionary history of Coffea. Botanical Journal of the Linnean Society, v. 167, n. 4, p. 357-377, 2011.

DE BRUYN Florac, et al. Characterization of organic compounds by gas chromatography. Part 1 . Retention indices of aliphatic halides, alcohols, aldehydes and ketones. Helvetica Chimica Acta, v. 41, p. 1915-1932, 1958.

DEBONA DG, et al. Sensory analysis and mid-infrared spectroscopy for discriminating roasted specialty coffees. Coffee Science, v. 16: e161878, 2021. https://doi.org/10.25186/.v16i.1878.

INCAPER. Café sustentável. Incaper em revista, v. 1, n. 1, p. 50-51, 2012.

LAWLESS, Harry T.; HEYMANN, Hildegarde. Physiological and psychological foundations of sensory function. In: Sensory evaluation of food. Springer New York, p. 19-56, 2010.

LEITE, Irã Pereira. Influência do local de cultivo e do tipo de colheita nas características físicas, composição química do grão e qualidade do café (Coffea arabica L.). Dissertação (Mestrado em Ciências dos Alimentos) - Escola 
Superior de Agricultura de Lavras. Lavras, 131p 1991.

MACHADO, Jéssica Louzada. Perfil químico e sensorial de grãos de diferentes genótipos de Coffea canephora. Universidade

Federal Do Espírito Santo, 78 p., 2019.

MACHADO. Jéssica Louzada et al.

Evaluation of genetic divergence of Coffee genotypes using the volatile compounds and sensory attributes profile. Food

Science, in press.

MALTA, Marcelo Ribeiro et al.

Discrimination of genotypes coffee by chemical composition of the beans:

Potential markers in natural coffees. Food

Research International, v. 134, p. 109219, 2020.

https://doi.org/10.1016/j.foodres.2020.109 219.

MARCOLAN, Alaerto Luiz; ESPINDULA, Marcelo Curitiba. Café na Amazônia. 1. ed. Brasília, DF: EMBRAPA Rondônia, 2015.

MARQUES, João Paulo. Relação entre o processamento de pós-colheita e atributos químicos e sensoriais de cafés de três locais de Minas. Gerais. Universidade José do Rosário Vellano (Unifenas), p. 53, 2017.

MARTINS, Ana Luiza. História do café. Editora Contexto, 2. Ed. São Paulo, SP, p. 130, 2012.. ISBN 978-85-7244-527-6.

MONDELLO, Luigi et al. Reliable characterization of coffee bean aroma profiles by automated headspace solid phase microextraction-gas chromatographymass spectrometry with the support of a dual-filter mass spectra library. Journal of Separation Science, v. 28, p. 1101-1109, 2005.

MOREIRA, Ricardo Felipe Alves; TRUGO, Luiz Carlos; DE MARIA, Carlos Alberto Bastos. Componentes voláteis do café torrado: parte II: compostos alifáticos, alicíclicos e aromáticos. Química Nova, São Paulo, v. 23, n. 2, p. 195-203, 2000.

OKSANEN, JARI et al. Vegan: Community Ecology Package. https://CRAN.Rproject. org/package=vegan, 2020.
PEREIRA Lucas Louzada et al. (2018). Propositions on the Optimal Number of QGraders and R-Graders. Journal of Food Quality, v. 7, p. ID 3285452. https://doi.org/10.1155/2018/3285452

PEREIRA Lucas Louzada et al. New propositions about coffee wet processing: Chemical and sensory perspectives. Food Chemistry, v. 310, p. 125943, 2020. https://doi.org/10.1016/j.foodchem.2019.1 25943

PEREIRA Priscila Vargas et al. (2021). Microbial diversity and chemical characteristics of Coffea canephora grown in different environments and processed by dry method. World Journal of Microbiology and Biotechnology, v. 37, p. 51. https://doi.org/10.1007/s11274-02103017-2

PEREIRA, Lucas Louzada et al. Tamanho ótimo do número de Q-Graders de café com uso do protocolo SCAA. Simpósio Internacional de Ingeniería Industrial: Actualidad y NuevasTendencias, 9., 2016.

PEREIRA, Rosemary Gualberto Fonseca Alvarenga. Efeitos da inclusão de grãos defeituosos na composição química e qualidade do café (Coffea arábica L.) "Estritamente Mole”. Tese (Doutorado) Programa de Pós-Graduação em Ciências dos Alimentos, Universidade Federal de Lavras, 96p. 1997.

QU, Chen et al. (2021). Predicting Kováts Retention Indices Using Graph Neural Networks. Journal of Chromatography A, v. 1646, p. 462100. https://doi.org/10.1016/j.chroma.2021.462 100.

R CORE TEAM. "R: A Language and Environment for Statistical Computing." https://www.Rproject.org/.2021.

RIBEIRO, J. S.; et al. Prediction of sensory properties of Brazilian Arabica roasted coffees by headspace solid phase microextraction-gas chromatography and partial least squares. Analytica Chimica Acta, Amsterdam, v. 364, p.172-179, 2009. 
ROCHA, Rodrigo Barros et al. Melhoramento de Coffea canephora - considerações e metodologias. MARCOLAN, A. L.; ESPINDULA, M. C. Café na Amazônia. 1. ed. Brasília, DF:EMBRAPA Rondônia, 2015.

SANZ, Cristina et al. Comparison of potent odorants in a filtered coffee brew and in an instant coffee beverage by aroma extract dilution analysis (AEDA). European Food Researchand Technology, v. 214, p. 299302, 2002.

SILVA, Érico Farias da. Mercado internacional do café: contexto histórico, cenário atual e algumas perspectivas.

Universidade Federal do Rio Grande do Sul, 57p, 2017.

SOUZA, Flávio de França et al. Aspectos gerais da biologia e da diversidade genética de Coffea canephora. In: MARCOLAN, A.L, ESPÍNDULA, M.C. Café na Amazonia. Embrapa, Brasília, DF, 2015, 474 p.

SPECIALTY COFFEE ASSOCIATION OF AMERICA (SCAA). Metodologia SCAA de avaliação de cafés especiais: Guia rápido - Green Coffee, 2009. Disponível em: <http://coffeetraveler.net/wpcontent/files/903

TANG, Vivien Chia Yen et al. Effect of solid-state fungal fermentation on the nonvolatiles content and volatiles composition of Coffea canephora (Robusta) coffee beans. Food Chemistry, v. 337, p. 128023, 2021.

TAUNAY, Affonso de E. Pequena História do café no Brasil (1727- 1937). Rio de Janeiro: Edição do Departamento Nacional do Café, 1945.

TOCI, Aline T.; FARAH, Adriana. Volatile fingerprint of Brazil and effective coffee seeds: corroboration of potential marker compounds and identification of new low quality indicators. Food Chemistry, v. 153, p. 298-314, 2014.

Uganda Coffee Development Authority (UCDA). Uganda Coffee Development Authority. Annual Report, 2012.

WANG, Yanbing et al. Chemical ingredients characterization basing on $1 \mathrm{H}$ NMR and
SHS-GC/MS in twelve cultivars of Coffea arabica roasted beans. Food Research International, v. 147, p. 110544, 2021. WICKHAM, Hadley. ggplot2 Elegant

Graphics for Data Analysis, v. . 35, 2009. https://doi.org/10.1007/978-0-387-981413. 